OPEN ACCESS

Edited by:

Tauqeer Hussain Mallhi,

Al Jouf University, Saudi Arabia

Reviewed by:

Gönül Tanir,

Dr. Sami Ulus Child Health and Diseases Training and Research

Hospital, Turkey

Chris Mast,

Merck, United States

Louisa Pollock

Royal Hospital for Children,

United Kingdom

*Correspondence: Leo Markovinović

fijufiju@gmail.com

Specialty section: This article was submitted to Pediatric Infectious Diseases, a section of the journal Frontiers in Pediatrics

Received: 20 April 2020 Accepted: 20 November 2020 Published: 21 December 2020

Citation:

Markovinović L, Knezović I, Kniewald T, Stemberger Marić L

Trkulja V and Tešović G (2020)

Enteroadsorbent Polymethylsiloxane Polyhydrate vs. Probiotic Lactobacillus reuteri DSM 17938 in the Treatment of Rotaviral Gastroenteritis in Infants and Toddlers, a Randomized Controlled Trial. Front. Pediatr. 8:553960. doi: 10.3389/fped.2020.553960

\section{Enteroadsorbent Polymethylsiloxane} Polyhydrate vs. Probiotic Lactobacillus reuteri DSM 17938 in the Treatment of Rotaviral Gastroenteritis in Infants and Toddlers, a Randomized Controlled Trial

\author{
Leo Markovinović ${ }^{1 *}$, Ivica Knezović ${ }^{1}$, Tihana Kniewald ${ }^{1}$, Lorna Stemberger Marić ${ }^{1,2}$, \\ Vladimir Trkulja ${ }^{3}$ and Goran Tešović ${ }^{1,3}$ \\ 1 University Hospital for Infectious Diseases "Dr. Fran Mihaljević", Zagreb, Croatia, ${ }^{2}$ School of Dental Medicine, University of \\ Zagreb, Zagreb, Croatia, ${ }^{3}$ School of Medicine, University of Zagreb, Zagreb, Croatia
}

Purpose: The aim of this study was to compare two adjunct therapies in the treatment of childhood rotavirus gastroenteritis (RVGE). We compared the recommended treatment, probiotic Lactobacillus reuteri DSM 17938 (BioGaiaß), vs. a novel treatment, enterosorbent polymethylsiloxane polyhydrate (Enterosgel@).

Methods: This was an open-label, randomized, clinical controlled trial at the University Hospital for Infectious Diseases (UHID) in Zagreb, Croatia. A total of 149 children aged 6-36 months with acute rotaviral gastroenteritis over a period of $<48 \mathrm{~h}$, with no significant chronic comorbidity, were randomized to receive the standard therapy with $L$. reuteri DSM 17938 (hereafter L. reuteri) or polymethylsiloxane polyhydrate (hereafter PMSPH) therapy, during 5 days. The primary end point was time to recovery in days in both groups. The recovery was defined as absence of fever and vomiting and either the first firm stool, absence of stool for more than $24 \mathrm{~h}$, or return of usual bowel habit.

Results: A total of 75 children were randomized into the $L$. reuteri group and 74 were randomized into the $\mathrm{PMSPH}$ group; after excluding missing data, the data from 65 children in each group were analyzed. There was no significant difference in the treatment efficacy between the two regimens with an estimated median time of recovery of 6 days in both groups $(p=0.754)$. No significant side effects were observed in either group.

Conclusion: Novel enterosorbent PMSPH had a similar efficacy to probiotic L. reuteri in the treatment of rotaviral gastroenteritis in preschool children.

Clinical Trial Registration: ClinicalTrials.gov Identifier: NCT04116307 [October 3, 2019] (retrospectively registered). https://clinicaltrials.gov/show/NCT04116307.

Keywords: gastroenteritis, children, polymethylsiloxane polyhidrate, Lactobacillus reuteri DSM 17938, rotavirus 


\section{THE STUDY}

\section{Background}

Rotavirus is the most frequent cause of acute gastroenteritis (AGE) in preschool children in countries without universal infant vaccination against rotavirus $(\mathrm{RV})(1,2)$. A similar situation can be observed in Croatia since universal vaccination against $\mathrm{RV}$ is still not part of the national immunization schedule. Health insurance covers only the vaccination of the population at risk, such as children with heart defects, chronic kidney and liver diseases, metabolic illnesses, and severe brain damage. The reasons for such practice are purely financial. Based on the number of vaccines sold over the counter, we can estimate that only $10 \%$ of children have been vaccinated against RV in Croatia in the last few years (3).

The pathogenesis of RV infection consists of direct damage of duodenal enterocytes by the virus, which causes malabsorption, and the toxic effect of viral nonstructural protein 4 (NSP4), which causes increased fluid secretion, damage to tight junctions, and increased intestinal motility (4). Clinical manifestations of RVGE include all three symptoms of AGE - vomiting, fever, and diarrhea, which amplify the loss of water and contribute to the severity of the disease. There is no causative therapy for the treatment of RV infection. The mainstay of treatment is rehydration and antipyretics. Children who do not tolerate oral rehydration therapy (ORT) could be given intravenous solutions of glucose and electrolytes. Several studies have shown that probiotics (including $L$. reuteri) shorten the period of diarrhea in children and reduce the need for hospitalization (5-9). The precise mechanism of action of probiotics is not fully understood. It is known that modification of the gut microbiota can have beneficial effects such as competition with pathogens for food and adherence points, strengthening of the gut epithelial barrier, and modulation of the gut immune system (10). According to the Cochrane Library metaanalysis, probiotic treatment for acute infectious diarrhea shows clear benefits in the shortening of diarrhea duration and reduction of stool frequency (11). Thus, the guidelines of the European Society for Pediatric Gastroenterology, Hepatology and Nutrition (ESPGHAN) and the European Society for Pediatric Infectious Diseases (ESPID) suggest that some probiotics can be considered for the treatment of gastroenteritis. These include the first-line probiotics Lactobacillus rhamnosus GG (LGG) and Saccharomyces boulardii. Lactobacillus reuteri DSM 17938 and Lactobacillus acidophilus LB are also included in the list of recommended strains (12).

Regarding enterosorbent use as an adjunctive therapy for AGE, ESPGHAN/ESPID guidelines only mention diosmectite, a natural mineral clay, to be considered as a therapeutic option (12). Another therapeutic enterosorbent, with proven efficacy

\footnotetext{
Abbreviations: AE, adverse events; AGE, acute gastroenteritis; CFU, colonyforming units; ESPGHAN, European Society for Pediatric Gastroenterology, Hepatology and Nutrition; ESPID, European Society for Pediatric Infectious Diseases; ITT, intention-to-treat; LGG, Lactobacillus rhamnosus GG; NSP4, nonstructural protein 4; ORT, oral rehydration therapy; PMSPH, polymethylsiloxane polyhydrate; RV, rotavirus; RVGE, rotavirus gastroenteritis; UHID, University Hospital for Infectious Diseases.
}

in the clinical setting, is the medical device PMSPH (13-16). Its effectiveness is similar to probiotics although the mode of action is different. PMSPH possesses a preferential adsorption capacity for larger molecules, such as bacterial toxins and protein degradation products. It also forms a thin layer over the mucosal surface and thus protects it from various damaging factors (17).

The aim of this randomized non-inferiority study was to compare the efficacy, tolerability, and safety of two adjunct therapies: probiotic $L$. reuteri vs. enterosorbent $\mathrm{PMSPH}$, in the treatment of RVGE in childhood.

\section{METHODS}

We carried out a prospective, open-label, randomized, controlled trial from January 1, 2013, until May 31, 2017. The study participants were children treated for RVGE at the outpatient department or hospital ward of the University Hospital for Infectious Diseases (UHID), Zagreb. The inclusion criteria were as follows: age 6-36 months, proven RV infection, duration of illness $<48 \mathrm{~h}$ before the first visit, and signed written consent by parents or caregivers for participation in the study. The exclusion criteria were as follows: vaccination against RV, previous laboratory-confirmed RV infection in patient's history, and some chronic illnesses or conditions that could influence the course of RV infection (food allergy, malabsorption, liver or pancreatic failure, chronic heart disease, or immune deficiencies). A small number of children (six) started taking probiotics prior to the inclusion into our study for various reasons. If they had taken probiotics different from $L$. reuteri they were excluded from the study. They were also excluded if they had taken L. reuteri and were randomized into the PMSPH group. Infants that had taken $L$. reuteri in a small dose for the treatment of previous infant colic were not excluded. Three children were included in the probiotic group, who already started taking L. reuteri for infant colic.

All parents/guardians were informed about the survey, both in direct contact with the investigator and by a written letter containing all the details about the study. If they agreed, they signed the informed consent. Children meeting inclusion criteria, whose parents/guardians provided a signed written informed consent, were randomized to $L$. reuteri or PMSPH groups. To achieve the balance between two groups, we used permutated block randomization (random block size 2-6). The randomization list was kept in a sealed envelope by the Department Head Nurse not involved in the patient enrollment or provision of care. Parents/guardians were given a simple daily diary to record their child's symptoms. The diary recorded the following symptoms: frequency of stools, frequency of vomiting, and body temperature of the subject for 8 days from the beginning of the symptoms. Stools were recorded as firm $(\bullet)$, semi-liquid $(\odot)$, and liquid (o).

The comparative study intervention was the class IIa medical device PMSPH. It was administered orally as recommended in the Instructions for Use, $3 \times 10 \mathrm{~g}$ dissolved in the same amount 


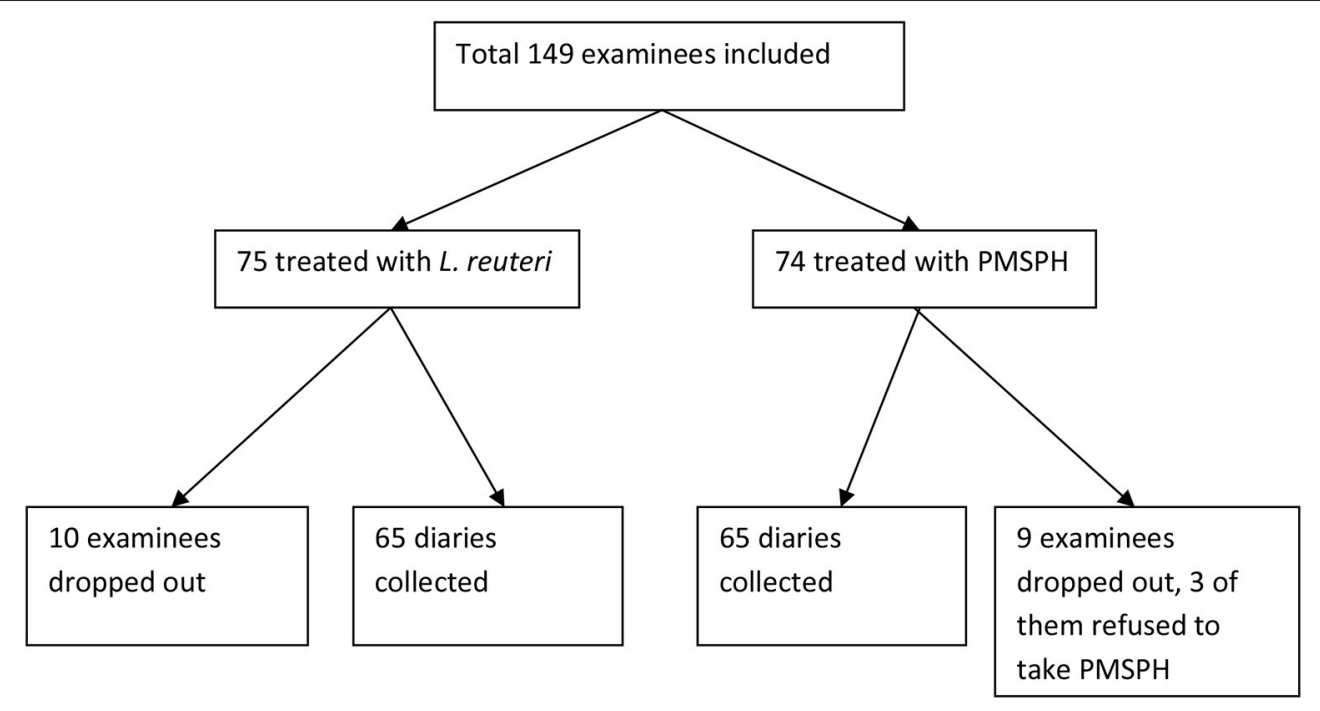

FIGURE 1 | CONSORT flow diagram of the randomized trial of children with rotavirus infection.

of water for the first two days, and $3 \times 5 \mathrm{~g}$ for the remaining three days. The control treatment was probiotic L. reuteri, which was given at a dose of $1.2 \times 10^{9}$ colony-forming units (CFU) per day. All subjects also received parenteral rehydration using isotonic intravenous solutions of $2.5 \%$ glucose/half normal saline or pure normal saline. Parallel to intravenous rehydration, or soon after the child's status improved, all children also were given ORT, which is a standard treatment for gastroenteritis of any cause.

The three main symptoms that constitute the clinical presentation of RV infection were measured on a daily basis: fever, vomiting, and liquid stools. At first visit, parents/caregivers were shown how to measure and record symptoms in the diary, and subsequently recorded symptoms themselves. The symptoms were recorded during the 8 days from the beginning of the illness, after which parents/caregivers returned diaries to the site by mail.

The majority of study participants were examined at the UHID pediatric emergency room and admitted to the hospital/day hospital. Some of the patients already hospitalized for other reasons were also included in the study after they acquired nosocomial RV infection. During the hospital stay, hematologic and biochemical tests were performed as well as a rapid stool test for RV and adenovirus (Rota-AdenoGnost test ${ }^{\circledR}$, BioGnost, Hannover, Germany). For all patients included in the study, we routinely performed complete blood count, blood glucose, urea \pm creatinine, $\mathrm{Na}, \mathrm{K}, \mathrm{Cl}$, aspartate aminotransferase (AST), alanine aminotransferase (ALT), and stool culture. For some patients, we also conducted additional tests such as acidbase balance, urinalysis, abdominal ultrasound, blood, and/or urine culture.

The subjects were also scored according to the Vesikari and Clark Clinical Severity Scoring System, which is used as a standardized methodology primarily across rotavirus vaccine studies $(18,19)$.
After data collection, the statistical analysis was performed using SAS for Windows 9.4 software licensed at the Zagreb University School of Medicine.

Primary analysis was conducted on a per-protocol analysis. The primary end point was time to recovery in a number of days. The primary end point was analyzed by KaplanMeier and Cox proportional hazards modeling. Recovery was defined as absence of fever and vomiting and (a) first firm stool; (b) absence of stool (and other symptoms) for more than $24 \mathrm{~h}$; or (c) returning of the usual bowel habit. It is known that infants can have mushy stools each day during their healthy period.

Study intervention efficacy was tested on three levels: duration of illness, length of hospital stay, and the total number of stools during the defined period ( 8 days). For the analysis of duration of illness and length of hospital stay, a proportional hazard regression method was used, while for the total number of stools, the Mann-Whitney test was used. The demographic and epidemiological data were analyzed by descriptive statistics methods.

The primary outcome measure of the efficacy was the duration of symptoms measured as time to recovery in days. In a large study comparing several probiotics in 600 children with AGE, the probiotic $L$. casei $(N=100)$ had a median time to recovery of 3.25 days with a range of $2-8$ days (20). The sample size calculation for a statistical power of $80 \%$ for a non-inferiority test according to given criteria (primary outcome), data of the span, and median of the time to recovery taken from the literature (20) was converted to mean value and standard deviation (3.25 \pm 1 day) (21). With an assumption of the same deviation in the test group, for desired power of noninferiority, it was necessary to have 64 subjects per group (128 in total) (22).

Test treatment was considered non-inferior to control treatment if the upper limit of $97.5 \%$ confidence interval for 
TABLE 1 | Participant demographics and baseline data summarized by trial arm as analyzed.

\begin{tabular}{|c|c|c|}
\hline Variable & PMSPH $(n=65)$ & L. reuteri $(n=65)$ \\
\hline Age (months) & $17.4(6.4-35.8)$ & $15.3(6.3-35.9)$ \\
\hline \multicolumn{3}{|l|}{ CLINICAL CHARACTERISTICS } \\
\hline \multicolumn{3}{|c|}{ General condition at the beginning of the study } \\
\hline Good & $2(3.1 \%)$ & $1(1.5 \%)$ \\
\hline Critical & 0 & 0 \\
\hline \multicolumn{3}{|l|}{ Type of rehydration treatment } \\
\hline Exclusively oral & 0 & 0 \\
\hline Parenteral & $65(100 \%)$ & $65(100 \%)$ \\
\hline Vesikari score & $18(11-20)$ & $17(11-20)$ \\
\hline Gastrointestinal co-infection & $2(3.1 \%)$ & $0(0)$ \\
\hline Hospital-acquired infection & $6(9.2 \%)$ & $10(15.4 \%)$ \\
\hline \multicolumn{3}{|l|}{ LABORATORY DATA } \\
\hline C-reactive protein (mg/L) & $4.0(0.2-44.8)$ & $4.7(0.1-41.7)$ \\
\hline Total number of leukocytes $\left(\times 10^{9} / L\right)$ & $9.5(3.8-30.6)$ & $10.8(3.3-27.3)$ \\
\hline Percentage of neutrophils & $72(18-92)$ & $67(10-95)$ \\
\hline Blood urea (mmol/L) & $5.8(0.5-10.4)$ & $5.1(2.4-8.9)$ \\
\hline Sodium (mmol/L) & $138(131-144)$ & $138(132-145)$ \\
\hline Potassium (mmol/L) & $4.1(3.2-5.1)$ & $4.3(3.2-5.2)$ \\
\hline Chloride (mmol/L) & $102(96-111)$ & $103(96-113)$ \\
\hline Aspartate aminotransferase (IJ/L) & $50(19-72)$ & $46(22-71)$ \\
\hline
\end{tabular}

*Hospitalization means that the child stayed in the hospital continuously for longer than $24 \mathrm{~h}$.

${ }^{\star}$ Day hospital means that the child was admitted to the hospital, stayed in it for $<24 \mathrm{~h}$, and could be checked and treated in the hospital in the next few days, if required.

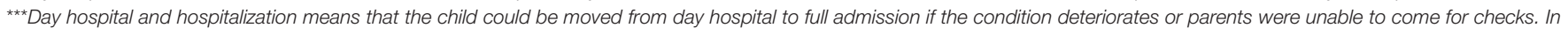
converse, a child who was initially hospitalized could be moved to day hospital once condition improved.

test control did not exceed 0.5 days (i.e., time to recovery with $T$ and $97.5 \%$ certainty was no longer than 0.5 days with control treatment).

\section{RESULTS}

The study CONSORT flow diagram (Figure 1) shows the progress of the study participants. Initially, altogether 149 children were enrolled, but only 130 sent back the diary. The data from 130 subjects, 65 from each group, were collected and analyzed.

Children who did not meet the inclusion criteria were discarded by the emergency department doctor, not by the investigator, so the exact number is unknown. The main reason for non-participation was parental refusal to participate and late arrival to the hospital (beyond $48 \mathrm{~h}$ after the onset of symptoms). The number of vaccinated children was negligible.
After assessing for eligibility, 149 subjects were randomized to either the L. reuteri or the PMSPH group. One hundred thirty subjects completed the study and were analyzed.

The demographic and clinical characteristics in both therapeutic groups were similar except for a somewhat higher frequency of respiratory symptoms or respiratory co-infection in the probiotic group that could influence hospital stay length (Table 1). It is important to emphasize that both groups of children were given ORT alongside test treatments.

The primary measure of efficacy was the time in days till recovery. Figure 2 summarizes cumulative achievement of recovery in the two therapeutic groups: all the children recovered at the latest by the eighth day since the onset of the illness with comparable dynamics in both groups. Kaplan-Meier estimation of the median of the time of recovery was 6 days in both groups.

Table 2 shows results of the univariate and multivariate comparison of the two treatments (treatment 1: L. reuteri; treatment 2: $\mathrm{PMSPH}$ ) according to the (current) risk of 


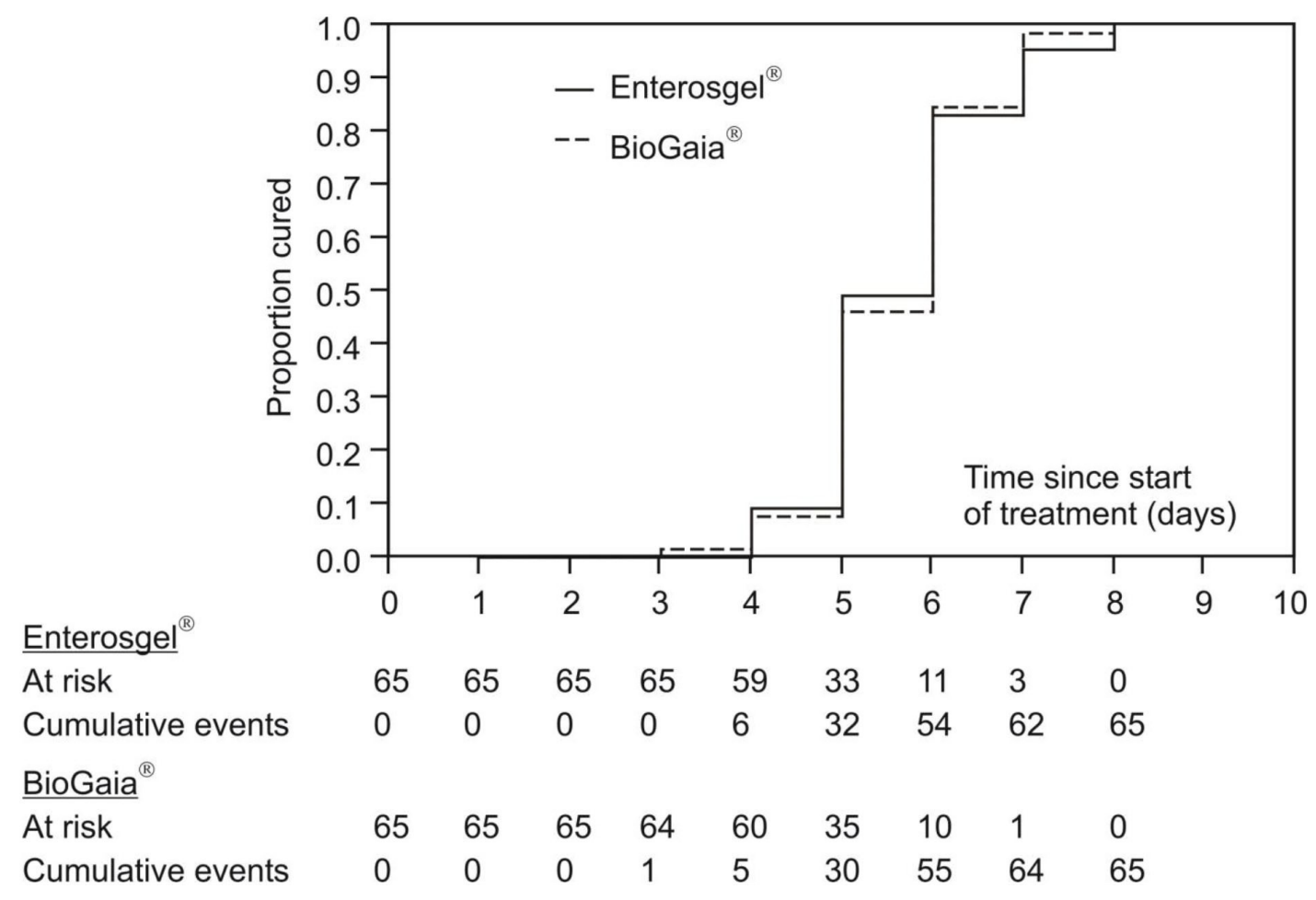

FIGURE 2 | Kaplan-Meier curves of cumulative recovery in the PMSPH (Enterosgel囚) and L. reuteri (BioGaia®) treatment groups for children with RVGE.

recovering from the rotavirus gastroenteritis (results of proportional hazards regression are condensed). Without any adjustment for any type of covariates (univariate model), current risk (probability) of recovery with PMSPH was not significantly different to that with $L$. reuteri $(\mathrm{HR}=0.94,95 \%$ CI $0.65-1.36$; $p=0.754)$. Comparable results were achieved for the two multivariate models-Model 1 in Table 2 ( $\mathrm{HR}=0.89,95 \%$ CI $0.60-1.32 ; p=0.567$ ) where, by the other covariates, Vesikari score was included and Model 2 in Table 2 (HR $=1.06$; 95\% CI $0.75-1.49 ; p=0.816$ ) where, by the other covariates, Clark's score was included (instead of Vesikari score).

The secondary measure of efficacy of the two treatments was the time from the start of study treatment to hospital discharge. Figure 3 summarizes the cumulative achievement of the cessation of hospital stay: all children were discharged by day 12 from the start of treatment, with dynamics completely comparable for the two treatments. The KaplanMeier estimation of the median time to hospital discharge was 3 days for both groups.

Table 3 summarizes the results of two multivariate models of proportional hazards regression, with identical variables to those in Table 2, except in this table, the time to discharge from hospital was analyzed. In both models, the first that includes the Vesikari score and the second that includes the Clark score, there was no significant difference between the two treatments according to the (current) risk of discharge from hospital: $\mathrm{HR}=1.17$ (95\% CI $0.80-1.72 ; p=0.419)$ and $\mathrm{HR}=1.25$ (95\% CI $0.86-1.83$; $p=0.239$ ).
Altogether, the statistical analyses of the length of hospital stay follow those of the primary outcome measure of treatment efficacy, which means no difference between the two treatments. Both analyses gave additional insights: the chances of recovery and hospital discharge were higher in older children and lower in the case of hospital-acquired RV infection, whereas the chances of both were lower with more severe disease according to the Vesikari score or Clark score for every two points gained. Model 2 also shows that chance of discharge from hospital was higher if the treatment was commenced later ( $42 \%$ relatively), possibly reflecting the partially spontaneous nature of recovery from the course of the illness.

The third measure of efficacy was the total number of stools during the illness (from the first symptom of RVGE till the end of survey, i.e., 8 days). In the group of children treated with PMSPH, the median number of stools was 23 (4-86), and in the group treated with $L$. reuteri, this was 25 (6-86); there was no significant difference between the treatment groups (95\% CI -6 to 3 ), $p=0.479$ (Mann-Whitney test).

The total number of adverse events (AEs) reported was one in the PMSPH group and none in the L. reuteri group. In the PMSPH group, one case of transient urticaria was reported, which coincided with the first dose of treatment. The urticaria was mild and self-limiting and did not recur with the second dose, and there was no need for cessation of treatment. This was likely not related to the intervention but to the condition itself. Three children refused to take PMSPH, which resulted in their exclusion from the study. This may have been the result 
TABLE 2 | The results of univariate and multivariate analysis of the two treatments according to the (current) risk of recovery from rotavirus gastroenteritis.

\begin{tabular}{|c|c|c|}
\hline & HR (95\% CI) & $\boldsymbol{P}$ \\
\hline \multicolumn{3}{|l|}{ Univariate model } \\
\hline PMSPH vs. L. reuteri & $0.94(0.65-1.36)$ & 0.754 \\
\hline \multicolumn{3}{|l|}{ Multivariate model 1} \\
\hline PMSPH vs. L. reuteri & $0.89(0.60-1.32)$ & 0.567 \\
\hline Age (for 2 months) ${ }^{\star}$ & $1.11(1.05-1.16)$ & $<0.001$ \\
\hline Boys (vs. girls) & $0.68(0.45-1.03)$ & 0.069 \\
\hline Hospital infection (vs. community-acquired infection) & $0.07(0.03-0.16)$ & $<0.001$ \\
\hline Gastrointestinal co-infection (vs. no co-infection) & $0.49(0.15-1.63)$ & 0.242 \\
\hline Day of illness at the beginning of treatment (for 1 day) & $1.30(0.95-1.78)$ & 0.102 \\
\hline Vesikari score (for two points) ${ }^{\star \star}$ & $0.49(0.38-0.63)$ & $<0.001$ \\
\hline \multicolumn{3}{|l|}{ Multivariate model 2} \\
\hline PMSPH vs. L. reuteri & $1.06(0.75-1.49)$ & 0.816 \\
\hline Age (for 2 months) & $1.07(1.03-1.12)$ & 0.002 \\
\hline Boys (vs. girls) & $0.71(0.48-1.04)$ & 0.078 \\
\hline Hospital infection (vs. community-acquired infection) & $0.05(0.02-0.11)$ & $<0.001$ \\
\hline Gastrointestinal co-infection (vs. no co-infection) & $0.83(0.19-2.51)$ & 0.834 \\
\hline Day of illness at the beginning of treatment (for 1 day) & $1.42(1.02-1.95)$ & 0.036 \\
\hline Clark score (for two points) & $0.65(0.54-0.79)$ & $<0.001$ \\
\hline
\end{tabular}

${ }^{\star}$ For every 2 months, the chance of recovery was higher by $11 \%$.

${ }^{\star \star}$ For every two points, the chance of recovery was reduced by $51 \%$.

${ }^{\star \star \star}$ For every two points, the chance of recovery was reduced by $35 \%$.

of PMSPH palatability or the relatively larger volume of the treatment $(20-30 \mathrm{ml})$ compared to only 20 drops (ca. $1 \mathrm{ml})$ of L. reuteri.

\section{DISCUSSION}

Due to the lack of universal RV vaccination in the national immunization program, RVGE is still one the most common reasons for pediatric emergency department visits in Croatia. The mainstay of treatment of RVGE is ORT. However, children often refuse to drink ORT, which leads to prostration and serious concern of parents/caregivers. In that case, the solution can be short-term intravenous parenteral rehydration. Worried parents often ask for some kind of adjunct therapy to shorten the duration of illness. In our study, we compared two such adjunct therapies for the treatment of RVGE.

\section{Principal Findings}

This is the first study comparing possible therapeutic impact of the novel enterosorbent (PMSPH) with the recommended (probiotic) treatment of RVGE in children. The study showed that for the duration of illness, the length of hospital stay, and the total number of liquid stools, the results did not differ between the PMSPH and probiotic groups during the testing period. The study also confirmed good safety profile of the PMSPH, as demonstrated by the number of AEonly one case of mild urticaria (most likely related to the underlying condition).

\section{The Idea of the Study and the Comparison With Other Studies}

When the new medical device PMSPH entered the Croatian market (2012), the idea to compare it with the probiotic treatment for RV was conceived. Compared with activated charcoal, PMSPH is a more potent absorbent in its binding ability toward high-molecular-weight compounds such as proteins and bacterial endotoxins $(13,23)$.

L. reuteri was chosen as a comparator because it was the first approved probiotic for the treatment of diarrhea in infants. L. reuteri was given in triple recommended dose because some studies showed a correlation between larger doses and a shorter duration of diarrhea, and we intended to maximize the probability of treatment efficacy (24).

Children aged 6-36 months were selected as it was most likely to be their first RV infection and consequently a more severe course of the disease. Children vaccinated against RV were excluded along with previous history of RV illness and children with chronic comorbidities or immune deficiencies, to eliminate any condition that could impact the disease course.

The children in both groups were given tested adjunct therapies alongside ORT or, initially, alongside intravenous rehydration fluids.

Our study did not show the advantage of either treatments as there was no significant difference in the duration of symptoms, with the median duration of symptoms till recovery at 6 days $(\mathrm{HR}=0.94,95 \% \mathrm{CI} 0.65-1.36 ; p=0.754)$. Search of the literature shows quite wide variations in the reported duration of RV illness without any intervention. In the study by Uhnoo et al. (25), the symptoms of RVGE in children lasted for 5.9 days. A second 


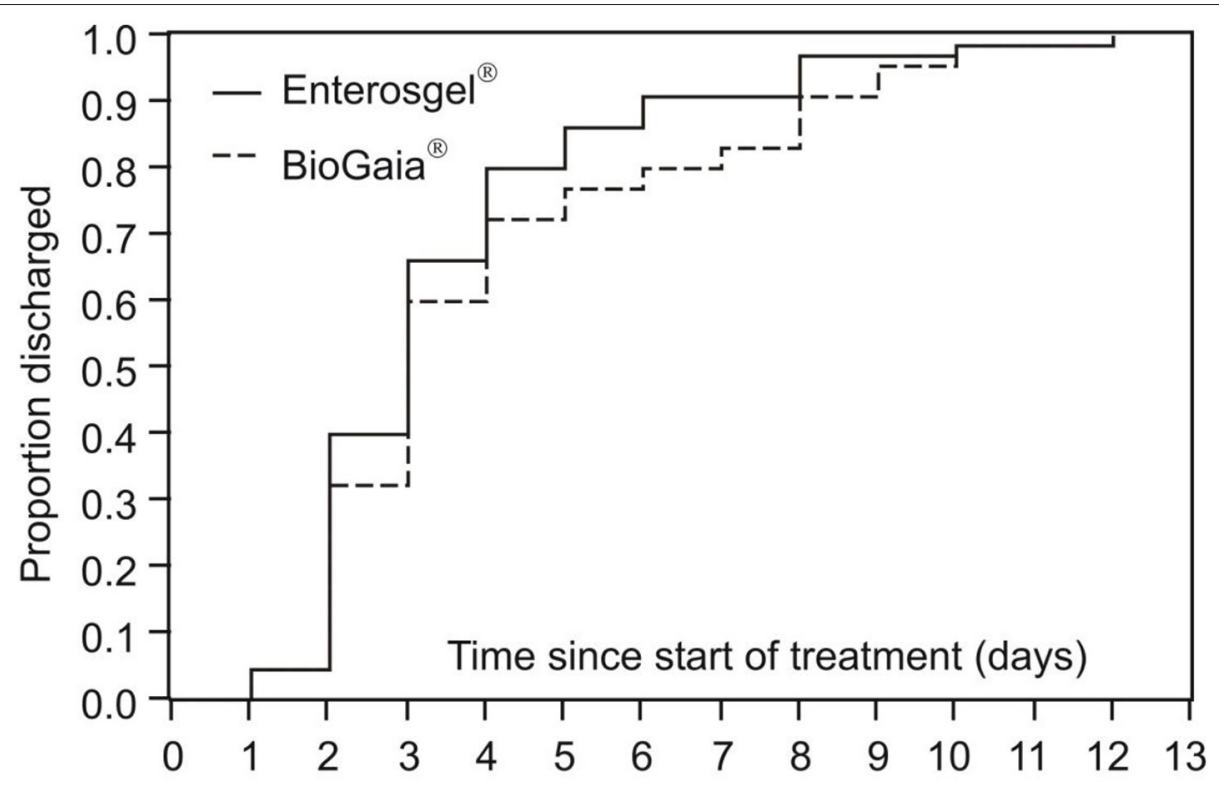

Enterosgel $^{\circledR}$

At risk

Cumulative events

BioGaia $^{\circledR}$

At risk

Cumulative events

\section{$\begin{array}{lllllllllllll}65 & 62 & 39 & 22 & 13 & 9 & 6 & 6 & 2 & 2 & 1 & 1 & 0\end{array}$ \\ $\begin{array}{lllllllllllll}0 & 3 & 26 & 43 & 52 & 56 & 59 & 59 & 63 & 63 & 64 & 64 & 65\end{array}$}

$$
\begin{array}{lllllllllllll}
65 & 62 & 44 & 26 & 18 & 15 & 13 & 11 & 6 & 3 & 1 & 1 & 0 \\
0 & 3 & 21 & 39 & 47 & 50 & 52 & 54 & 59 & 62 & 64 & 64 & 65
\end{array}
$$

FIGURE 3 | Kaplan-Meier curves of cumulative hospital discharge for the PMSPH (Enterosge|®) and L. reuteri (BioGaia®) treatment groups for children with RVGE.

study claimed the duration of symptoms of 4-8 days (26), while a third found that symptoms could last from 2 to 22 days (27). Considering these conflicting data, it is difficult to ascertain if there is any additional benefit to either intervention, if rotavirus illness can last for 5.9 days. However, a Cochrane analysis of 63 trials analyzing probiotic efficacy in the treatment of acute infectious diarrhea (of these, 56 trials recruited infants and young children) showed a mean shortening of diarrhea of $25 \mathrm{~h}$ and one stool less on day 2 from starting therapy. Of course, results vary between studies depending on the subject age (children vs. adults), the country where the study took place (developed country vs. developing country), type of probiotic/s, or the type of causative agent of diarrhea (11).

Many studies have shown beneficial effects of probiotics on the course of acute infectious diarrhea, shortening duration of symptoms, reducing the number of liquid stools, and reducing hospital stay (5-9). Data relating to probiotic treatment efficacy are quite varied. Canani et al. compared five best-selling probiotics in Italy, with $\sim 100$ participants per group, who received either placebo (simple oral rehydration solution) or a probiotic. The study showed that only probiotics that contained LGG or a mix of four strains (L. delbrueckii var. bulgaricus, L. acidophilus, S. thermophilus, and B. bifidum) obtained a statistically significant benefit, resulting in shortening of symptom duration and reducing daily stool output. Interestingly, the $S$. boulardii probiotic recommended by ESPGHAN/ESPID did not obtain an advantage over placebo (20). Other studies did not show any beneficial effects of LGG $(28,29)$ or the drug racecadotril (30), also suggested by ESPGHAN/ESPID to be considered in the treatment of childhood diarrhea. Recent investigation of the treatment efficacy of $L$. reuteri in AGE showed failure to shorten symptoms duration, but had some effectiveness in the reduction of hospital stay (31). Taking into account many recent studies, the ESPGHAN workgroup has published new clinical guidelines for using probiotics in the treatment of AGE. The recommended probiotics remain the same (with the exception of L. acidophilus LB, which is replaced with a combination of $L$. rhamnosus 19070-2 and L. reuteri DSM 12246). However, the recommendation grade determined from the current evidence changed from strong to weak for all four recommended probiotics (32). Considering the similar, relatively high price of both $L$. reuteri and $\mathrm{PMSPH}$, which is about 20 euros, and only minor benefit, we can question the reason for the recommendation of these adjunct therapies in low-income countries.

The second measure of efficacy was the duration in days spent in the hospital/day hospital visits. This parameter provides further evidence about possible influence of the treatment on 
TABLE 3 | The results of the multivariate comparison of the two treatments according to the (current) risk of hospital discharge of children with RVGE.

\begin{tabular}{llc}
\hline & HR (95 \% Cl) & $P$ \\
\hline Multivariate model 1 & & \\
PMSPH vs. L. reuteri & $1.17(0.80-1.72)$ & 0.419 \\
Age (for 2 months) & $1.07(1.02-1.11)$ & 0.005 \\
Boys (vs. girls) & $0.67(0.46-0.99)$ & 0.041 \\
Hospital infection (vs. community-acquired infection) & $0.07(0.03-0.15)$ \\
Gastrointestinal co-infection (vs. no co-infection) & $0.49(0.11-1.40)$ \\
Day of illness at the beginning of treatment (for 1 day) & $1.30(0.95-1.79)$ \\
Vesikari score (for two points) & 0.001 \\
Multivariate model 2 & $0.82(0.74-0.92)$ \\
PMSPH vs. L. reuteri & & 0.242 \\
Age (for 2 months) & $1.25(0.86-1.83)$ \\
Boys (vs. girls) & $1.07(1.03-1.12)$ \\
Hospital infection (vs. community-acquired infection) & $0.71(0.48-1.04)$ \\
Gastrointestinal co-infection (vs. no co-infection) & $0.05(0.02-0.11)$ \\
Day of illness at the beginning of treatment (for 1 day) & $0.83(0.19-2.51)$ \\
Clark score (for two points) & $1.42(1.02-1.95)$ \\
\hline
\end{tabular}

*For every two points, the chance for discharge from hospital was reduced by $18 \%$.

${ }^{\star *}$ For every two points, the chance for discharge from hospital was reduced by $19 \%$.

the course of illness, although it is not a direct measure. Some children can have a longer hospital stay than RVGE symptoms (for example, children who were hospitalized for other reasons), or shorter (for example, children with benign course of the illness where brief hospitalization is sufficient). The length of hospital stay can be indicative of the entire disease course and the potential impact of the treatment. Rosenfeldt et al. showed a positive effect of probiotics ( $L$. reuteri + LGG) not only on the duration of symptoms but also on the shortening of hospital stay by $48 \%$ (33). They used preparations with a higher probiotic concentration $\left(10^{10}-10^{11} \mathrm{CFU} /\right.$ day) than this study. However, we were not able to show a significant difference in the hospital stay between the two treatment groups $(\mathrm{HR}=1.17$ 95\% CI $0.80-1.72 ; p=0.419$ with Vesikari score included and $\mathrm{HR}=1.25$ 95\% CI 0.86-1.83; $p=0.239$ with Clark score included).

The third measure of efficacy was the total number of liquid stools during the illness. As some adjunct therapies for infectious AGE, such as the antisecretory drug racecadotril, have an effect on reducing stool output, we also analyzed this parameter. This study found no significant difference in the number of stools between the two treatment groups (95\% CI -6 to $3, p=0.479$; Mann-Whitney test).

The study did not record any AEs of the two treatments, except mild urticaria in one patient, which coincided with the first dose of PMSPH. We took it into account albeit this was unlikely to be the possible side effect of PMSPH, having in mind that this could be the consequence of RV infection itself.

\section{STRENGTHS AND LIMITATIONS}

This study had a few limitations. The main limitation is the small sample size. Because of this, the safety profile of the new drug should be taken with some reservations. The second limitation is the study design itself, which was not a doubleblind placebo-controlled study but an open-label, randomized, controlled study. We could not conduct a blinded treatment because of the different physical characteristics of the treatments (drops vs. gel). The same applied to the absence of a placebo group. It would have been unethical to have an intervention placebo control group and would have hindered recruitment to the study as it would be difficult to persuade parents (or caregivers) to consent to the study, knowing that their child could be given no treatment.

Another limitation was the method of measurement of diarrhea symptoms as the number of liquid stools rather than mass or volume, which is a more proportional measure of water loss. This measure was recorded by parents, and measuring volume or mass of stool would have required obligatory (unnecessary) hospitalization for 7-8 days, which was viewed as unethical.

On the positive side, the study population was very homogeneous as all subjects had similar clinical characteristics, thus making the comparison of the treatment efficacy more reliable.

\section{CONCLUSION}

This randomized, open-label, clinical controlled trial showed no significant difference in the therapeutic efficacy between the new enterosorbent PMSPH and probiotic L. reuteri in the treatment of RVGE in children. The two treatments have similar excellent safety profiles in young aged children. As probiotics have some contraindications (congenital or acquired immune deficiencies, heart valve defects, and damaged bowel mucous membranes), PMSPH could be used in these instances as a safe and effective alternative to probiotics. In summary, the results of our study support the use of PMSPH as adjunct therapy alongside ORT in 
the management of RVGE in children aged 6 months and older. However, further investigations are recommended to define treatment efficacy in comparison to different treatment options and populations.

\section{DATA AVAILABILITY STATEMENT}

The original contributions presented in the study are included in the article/supplementary materials, further inquiries can be directed to the corresponding author/s.

\section{ETHICS STATEMENT}

The studies involving human participants were reviewed and approved by the ethical committee of the University Hospital for Infectious Diseases, Zagreb, Croatia. Written informed consent to participate in this study was provided by the participants' legal guardian/next of kin.

\section{REFERENCES}

1. World Health Organization. Diarrhoeal Disease. (2017). Available online at: http://www.who.int/mediacentre/factsheets/fs330/en/ (accessed April 12, 2018).

2. Parashar UD, Hummelman EG, Bresee JS, Miller MA, Glass RI. Global illness and deaths caused by rotavirus disease in children. Emerg Infect Dis. (2003) 9:565-72. doi: 10.3201/eid0905.020562

3. Croatian Institute of Public Health. Implementation of Immunization, Immunoprophylaxis and Chemoprophylaxis Program in Croatia in 2017 (2017). Available online at: https://www.hzjz.hr/wp-content/uploads/ 2017/05/PROVEDBENI-PROGRAM-II._2017.pdf (accessed November 20, 2020).

4. Dormitzer PR. Rotavirus. In: Bennett JE, Dolin R, Blaser MJ, editors. Mandell, Douglas, and Bennett's: Principles and Practice of Infectious Diseases. 9th ed. Philadelphia, PA: Churchill Livingstone (2020). p. 1983-96.

5. Isolauri E, Juntunen M, Rautanen T, Sillanaukee P, Koivula T. A human Lactobacillus strain (Lactobacillus casei sp strain GG) promotes recovery from acute diarrhea in children. Pediatrics. (1991) 88:90-7.

6. Shornikova AV, Casas IA, Isolauri E, Mykkänen $H$, Vesikari T. Lactobacillus reuteri as a therapeutic agent in acute diarrhea in young children. J Pediatr Gastroenterol Nutr. (1997) 24:399-404. doi: 10.1097/00005176-199704000-00008

7. Rosenfeldt V, Michaelsen KF, Jakobsen M, Larsen CN, Møller PL, Tvede M, et al. Effect of probiotic Lactobacillus strains on acute diarrhea in a cohort of nonhospitalized children attending day-care centers. Pediatr Infect Dis J. (2002) 21:417-9. doi: 10.1097/00006454-200205000-00013

8. Dinleyici EC, PROBAGE Study Group, Vandenplas Y. Lactobacillus reuteri DSM 17938 effectively reduces the duration of acute diarrhoea in hospitalised children. Acta Paediatr. (2014) 103:e300-5. doi: 10.1111/apa.12617

9. Francavilla R, Lionetti E, Castellaneta S, Ciruzzi F, Indrio F, Masciale A, et al. Randomised clinical trial: Lactobacillus reuteri DSM 17938 vs. placebo in children with acute diarrhoea-a double-blind study. Aliment Pharmacol Ther. (2012) 36:363-9. doi: 10.1111/j.1365-2036.2012.05180.x

10. Bermudez-Brito M, Plaza-Díaz J, Muñoz-Quezada S, Gómez-Llorente C, Gil A. Probiotic mechanisms of action. Ann Nutr Metab. (2012) 61:160-74. doi: 10.1159/000342079

11. Allen SJ, Martinez EG, Gregorio GV, Dans LF. Probiotics for treating acute infectious diarrhoea. Cochrane Database Syst Rev. (2010) 2010:CD003048. doi: 10.1002/14651858.CD003048.pub3

12. Guarino A, Ashkenazi S, Gendrel D, Lo Vecchio A, Shamir R, Szajewska $\mathrm{H}$, et al. European Society for Pediatric Gastroenterology, Hepatology, and Nutrition/European Society for Pediatric Infectious Diseases evidencebased guidelines for the management of acute gastroenteritis in children

\section{AUTHOR CONTRIBUTIONS}

LM and GT designed the study and participated in examinees' recruitment. IK and LS participated in examinees' recruitment. TK designed the database. VT made statistical analysis and interpreted the patient data. All authors contributed to the article and approved the submitted version.

\section{FUNDING}

The authors declare that this study received the investigational drugs free of charge from the drug distributors: Martifarm and Oktal-Pharma (distributors of Enterosgel) and Ewopharma (distributor of BioGaia drops). These companies were not involved in the study design, collection, analysis, interpretation of data, the writing of this article, or the decision to submit it for publication.

in Europe: update 2014. J Pediatr Gastroenterol Nutr. (2014) 59:132-52. doi: 10.1097/MPG.0000000000000375

13. Dzyublik IV, Shun'ko EE, Barbova AI. Application of Enterosgel for Treatment of the Rotavirus Infection in Neonates. Kiev: Shupik Kiev Medical Academy of Post-Graduate Education (1997).

14. Barbova AI. The sorption of human and animal rotaviruses by enterosgel. Mikrobiol Z. (1995) 57:52-7.

15. Sharonina LY, Aleksandrova VV, Gerashchenko VI, Ul'yanova IV, Bandina O. Experience of treatment of patients suffered from the rotavirus infection. In: Proceedings of the Research and Practice Conference "Infectious Diseases and new Technologies in Medicine". Lipetsk (1998).

16. Howell CA, Markaryan E, Allgar V, Kemppinen A, Khovanov A, Pandya P, et al. Enterosgel for the treatment of adults with acute diarrhoea in a primary care setting: a randomised controlled trial. BMJ Open Gastroenterol. (2019) 6:e000287. doi: 10.1136/bmjgast-2019-000287

17. Nikolaev VG. Enterosgel: a novel organosiliconenterosorbent with a wide range of medical applications. In: Mikhalovsky S, Khajibaev A, editors. Biodefence: Advanced Materials and Methods for Health Protection. Dordrecht: Springer (2011).

18. Ruuska T, Vesikari T. Rotavirus disease in Finnish children: use of numerical scores for clinical severity of diarrhoeal episodes. Scand J Infect Dis. (1990) 22:259-67. doi: 10.3109/00365549009027046

19. Clark HF, Borian FE, Plotkin SA. Immune protection of infants against rotavirus gastroenteritis by a serotype 1 reassortant of bovine rotavirus WC3. J Infect Dis. (1990) 161:1099-104. doi: 10.1093/infdis/161.6.1099

20. Canani RB, Cirillo P, Terrin G, Cesarano L, Spagnuolo MI, De Vincenzo A, et al. Probiotics for treatment of acute diarrhoea in children: randomised clinical trial of five different preparations. BMJ. (2007) 335:340. doi: 10.1136/bmj.39272.581736.55

21. Pudar Hozo S, Djulbegović B, Hozo I. Estimating sample mean and variance from the median, range and size of the sample. BMC Med Res Methodol. (2005) 5:13. doi: 10.1186/1471-2288-5-13

22. Chow SC, Shao J, Wang H. Sample Size Calculations in Clinical Research. New York, NY: Marcel Dekker (2003).

23. Howell CA, Mikhalovsky SV, Markaryan EN, Khovanov AV. Investigation of the adsorption capacity of the enterosorbent enterosgel for a range of bacterial toxins, bile acids and pharmaceutical drugs. Sci Rep. (2019) 9:5629. doi: 10.1038/s41598-019-42176-z

24. Shornikova AV, Casas IA, Mykkänen H, Salo E, Vesikari T. Bacteriotherapy with Lactobacillus reuteri in rotavirus gastroenteritis. Pediatr Infect Dis J. (1997) 16:1103-7. doi: 10.1097/00006454-199712000-00002

25. Uhnoo I, Olding-Stenkvist E, Kreuger A. Clinical features of acute gastroenteritis associated with rotavirus, enteric adenoviruses, and bacteria. Arch Dis Child. (1986) 61:732-8. doi: 10.1136/adc.61.8.732 
26. Gurwith M, Wenman W, Hinde D, Feltham S, Greenberg H. A prospective study of rotavirus infection in infants and young children. J Infect Dis. (1981) 144:218-24. doi: 10.1093/infdis/144.3.218

27. Wyatt RG, Yolken RH, Urrutia JJ, Mata L, Greenberg HB, Chanock RM, et al. Diarrhea associated with rotavirus in rural Guatemala: a longitudinal study of 24 infants and young children. Am J Trop Med Hyg. (1979) 28:325-8. doi: 10.4269/ajtmh.1979.28.325

28. Costa-Ribeiro H, Ribeiro TC, Mattos AP, Valois SS, Neri DA, Almeida $\mathrm{P}$, et al. Limitations of probiotic therapy in acute, severe dehydrating diarrhea. J Pediatr Gastroenterol Nutr. (2003) 36:112-5. doi: 10.1097/00005176-200301000-00021

29. Misra S, Sabui TK, Pal NK. A randomized controlled trial to evaluate the efficacy of Lactobacillus GG in infantile diarrhea. J Pediatr. (2009) 155:129-32. doi: 10.1016/j.jpeds.2009.01.060

30. Kang G, Thuppal SV, Srinivasan R, Sarkar R, Subashini B, Venugopal S, et al. Racecadotril in the management of rotavirus and non-rotavirus diarrhea in under-five children: two randomized, double-blind, placebo-controlled trials. Indian Pediatr. (2016) 53:595-600. doi: 10.1007/s13312-016-0894-0

31. Szymański H, Szajewska H. Lack of efficacy of Lactobacillus reuteri DSM 17938 for the treatment of acute gastroenteritis. A randomized controlled trial. Pediatr Infect Dis J. (2019) 38:e237-42. doi: 10.1097/INF.0000000000002355
32. Szajewska H, Canani RB, Guarino A, Hojsak I, Indrio F, Kolacek S, et al. Use of probiotics for the management of acute gastroenteritis in children: an update. J Pediatr Gastroenterol Nutr. (2020) 71:261-9. doi: 10.1097/MPG.0000000000002751

33. Rosenfeldt V, Michaelsen KF, Jakobsen M, Larsen CN, Møller PL, Pedersen $P$, et al. Effect of probiotic Lactobacillus strains in young children hospitalized with acute diarrhea. Pediatr Infect Dis J. (2002) 21:411-6. doi: 10.1097/00006454-20020500000012

Conflict of Interest: The authors declare that the research was conducted in the absence of any commercial or financial relationships that could be construed as a potential conflict of interest.

Copyright (C) 2020 Markovinović, Knezović, Kniewald, Stemberger Marić, Trkulja and Tešović. This is an open-access article distributed under the terms of the Creative Commons Attribution License (CC BY). The use, distribution or reproduction in other forums is permitted, provided the original author(s) and the copyright owner(s) are credited and that the original publication in this journal is cited, in accordance with accepted academic practice. No use, distribution or reproduction is permitted which does not comply with these terms. 